\title{
Gauge Theory and Gravity in the Loop Formulation ${ }^{1}$
}

\author{
Renate Loll \\ Center for Gravitational Physics and Geometry, Pennsylvania State University, \\ University Park, PA 16802-6300, USA
}

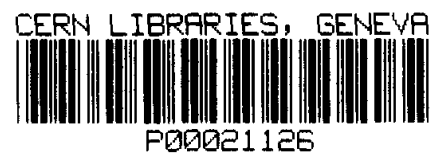

\section{Introduction}

This article introduces the basic notions and mathematical structures of the so-called loop approach to gauge theory and gravity. The two theories will be treated in parallel as far as this is possible. Emphasis will be put on those aspects of the loop formulation that make it conceptually different from other, local formulations. This contribution is meant to be complementary to other articles in this volume, but some overlap has been unavoidable to ensure a coherent presentation. Many more mathematical details and references for those who want to learn more about loops are contained in a related review (Loll 1993d). Another useful reference focussing on the gravitational application is the review article by Pullin (1993).

The following Section 2 summarizes the classical and quantum description of both gauge theory and gravity as theories on connection space, to set the stage for Sections 3-5 which deal with specific aspects of the loop formulation. In Section 3, I define paths, loops and holonomies, and briefly review past applications in physics. Section 4 is devoted to the identities satisfied by the holonomies and their traces, the latter also known as the Mandelstam constraints. It is shown how a group structure arises on the space of loops. Section 5 discusses the equivalence between the connection and the loop formulation which lies at the heart of the loop approach, and describes the content of the underlying reconstruction theorems. In Section 6, I introduce some ideas from lattice gauge theory which have proved useful in a regularized loop approach and enabled us to do certain calculations explicitly. To round off the discussion, Section 7 describes some features of quantized loop theories, and the final outlook contains a list of mathematical and physical inputs for the loop formulation which still require a more complete understanding.

1 To appear in the Proceedings of the Workshop on Classical and Quantum General Relativity, Bad Honnef, Germany, 1993, Lecture Notes in Physics, ed. H. Friedrich, Springer, Berlin 


\section{Yang-Mills theory and General Relativity as connection dynamics}

This section summarizes the Lagrangian and Hamiltonian formulation of both Yang-Mills gauge theory and gravity, defined on a four-dimensional manifold $M=\Sigma^{3} \times \mathbb{R}$ of Lorentzian signature. The gravitational theory will be described in the Ashtekar form, in which it most closely resembles a gauge theory. We will consider only the pure theories, without any matter coupling.

The classical actions defining these theories are (up to overall constants)

$$
S_{Y M}\left[{ }^{4} A\right]=-\int d^{4} x \operatorname{Tr} F_{\mu \nu} F^{\mu \nu}=-\int_{M} \operatorname{Tr}(F \wedge * F)
$$

and

$$
S_{G R}\left[{ }^{4} A, e\right]=\int d^{4} x e e_{I}^{\mu} e_{J}^{\nu} F_{\mu \nu}{ }^{I J}=\int_{M} \epsilon_{I J K L} e^{I} \wedge e^{J} \wedge F^{K L}
$$

for Yang-Mills theory and general relativity respectively. In (YM1), the basic variable $A$ is a g-valued connection one-form on $M$, where $G$ denotes the compact and semi-simple Lie structure group of the Yang-Mills theory (typically $G=S U(N)$ ), and $\mathbf{g}$ its Lie algebra. In (GR1), which is a first-order form of the gravitational action, $A$ is a self-dual, $s o(3,1)_{\mathbf{C}^{-}}$ valued connection one-form, and $e$ a vierbein, defining an isomorphism of vector spaces between the tangent space of $M$ and the fixed internal space with the Minkowski metric $\eta_{I J}$. The connection $A$ is selfdual in the internal space, $A_{\mu}^{M N}=-\frac{i}{2} \epsilon^{M N}{ }_{I J} A_{\mu}^{I J}$, with the totally antisymmetric $\epsilon$-tensor. As usual, $F$ denotes in both cases the curvature associated with the four-dimensional connection ${ }^{4} A$,

$$
F_{\mu \nu}(x)=\partial_{\mu} A_{\nu}(x)-\partial_{\nu} A_{\mu}(x)+\left[A_{\mu}(x), A_{\nu}(x)\right]
$$

Note that the action (GR1) has the unusual feature of being complex. I will not explain here why this still leads to a well-defined theory equivalent to the standard formulation of general relativity. Details may be found in the overview articles by Ashtekar $(1991,1993)$.

Our main interest will be the Hamiltonian formulations associated with (YM1) and (GR1). Assuming the underlying principal fibre bundles $P(\Sigma, G)$ is trivial, we can identify the relevant phase spaces with cotangent bundles over the affine spaces $\mathcal{A}$ of $g$-valued 
connections on the three-dimensional manifold $\Sigma$. (A connection space $\mathcal{A}$ is affine since only the difference, but not the sum of two of its elements lies again in $\mathcal{A}$; hence it is "not quite a vector space"). We assume the spatial manifold $\Sigma$ to be orientable and compact, in order not to have to worry about boundary terms. The cotangent bundle $T^{*} \mathcal{A}$ is coordinatized by canonically conjugate pairs $(A, \tilde{E})$, where $A$ is a g-valued, pseudo-tensorial one-form ("gauge potential") and $\tilde{E}$ a g-valued vector density ("generalized electric field") on $\Sigma$. We have $G=S U(N)$, say, for gauge theory, and $G=S O(3)_{\mathbb{C}}$ for gravity. The standard symplectic structure on $T^{*} \mathcal{A}$ is expressed by the fundamental Poisson bracket relations

$$
\left\{A_{a}^{i}(x), \tilde{E}_{j}^{b}(y)\right\}=\delta_{j}^{i} \delta_{a}^{b} \delta^{3}(x-y)
$$

In (2.2), $a$ and $b$ are three-dimensional spatial indices and $i$ and $j$ internal gauge algebra indices.

However, points in $T^{*} \mathcal{A}$ do not yet correspond to physical configurations, because both theories possess gauge symmetries, which are related to invariances of the original Lagrangians under certain transformations on the fundamental variables. At the Hamiltonian level, this manifests itself in the existence of so-called first-class constraints. They are sets $\mathcal{C}$ of functions $C$ on phase space, which are required to vanish, $C=0$, for physical configurations and at the same time generate transformation between physically indistinguishable configurations (for more details, see the article by Wipf in this volume). In our case, the first-class constraints are

$$
\mathcal{C}_{Y M}=\left\{D_{a} \tilde{E}^{a i}\right\}
$$

and

$$
\mathcal{C}_{G R}=\left\{D_{a} \tilde{E}^{a i}, C_{a} \equiv F_{a b}{ }^{i} \tilde{E}_{i}^{b}, C \equiv \epsilon^{i j k} F_{a b i} \tilde{E}_{j}^{a} \tilde{E}_{k}^{b}\right\}
$$

The physical interpretation of these constraints is as follows: the so-called Gauss law constraint $D_{a} \tilde{E}^{a i}=0$ restricts to configurations whose covariant divergence vanishes, and at the same time generates local gauge transformations in the internal space. The well-known YangMills transformation law corresponding to a finite group element $g \in \mathcal{G}$, the set of $G$-valued functions on $\Sigma$, is 


$$
(A, \tilde{E}) \mapsto\left(g^{-1} A g+g^{-1} d g, g^{-1} \tilde{E} g\right)
$$

The second set, $\left\{C_{a}\right\}$, of constraints in (GR2) generates three-dimensional diffeomorphisms of $\Sigma$ and the last expression, the so-called scalar or Hamiltonian constraint $C$, generates phase space transformations that are interpreted as corresponding to the time evolution of $\Sigma$ in $M$ in a space-time picture. Comparing (YM2) and (GR2), we see that in the Hamiltonian formulation, pure gravity may be interpreted as a Yang-Mills theory with gauge group $G=$ $S O(3)_{\mathbb{C}}$, subject to four additional constraints in each point of $\Sigma$. However, the dynamics of the two theories is different; we have

$$
H_{Y M}(A, \tilde{E})=\int_{\Sigma} d^{3} x\left(E_{a}^{i} E_{i}^{a}+B_{i}^{a} B_{a}^{i}\right)
$$

as the Hamiltonian for Yang-Mills theory (where for convenience we have introduced the generalized magnetic field $B_{i}^{a}:=-\frac{1}{2} \epsilon^{a b c} F_{b c i}$ ). Note the explicit appearance of a Riemannian background metric $g$ on $\Sigma$, to contract indices and ensure the integrand in (YM3) is a density. On the other hand, no such additional background structure is necessary to make the gravitational Hamiltonian well-defined. The Hamiltonian $H_{G R}$ for gravity is a sum of a subset of the constraints (GR2),

$$
H_{G R}(A, \tilde{E})=i \int_{\Sigma} d^{3} x\left(N^{a} F_{a b}{ }^{i} \tilde{E}_{i}^{b}-\frac{i}{2} N \epsilon^{i j k} F_{a b k} \tilde{E}_{i}^{a} \tilde{E}_{j}^{b}\right)
$$

and therefore vanishes on physical configurations. ( $N$ and $N^{a}$ in (GR3) are Lagrange multipliers, the so-called lapse and shift functions.) This latter feature is peculiar to generally covariant theories, whose gauge group contains the diffeomorphism group of the underlying manifold. A further difference is the need for a set of reality conditions for the gravitational theory, because we are using the complex coordinate $A$ on phase space.

The space of physical configurations of Yang-Mills theory is the quotient space $\mathcal{A} / \mathcal{G}$ of gauge potentials modulo local gauge transformations. It is non-linear and has non-trivial topology. By excluding the reducible connections from $\mathcal{A}$ (roughly speaking, these are the connections with some additional symmetry properties), and slightly restricting the gauge group $\mathcal{G}$, this quotient can be given the structure of an infinite-dimensional manifold for compact and semi-simple $G$ (Arms et al. 1981). The corresponding physical phase space is then its cotangent bundle $T^{*}(\mathcal{A} / \mathcal{G})$. Elements of $T^{*}(\mathcal{A} / \mathcal{G})$ are called the classical observables of the Yang-Mills theory. 
With Ashtekar's reformulation of general relativity, a remarkable unification has been achieved in the description of the fundamental interactions of physics, which are all described as theories on spaces of connections, subject to a local gauge principle. Moreover, a similar formulation exists for a whole class of so-called topological field theories, examples of which are Chern-Simons theory and $2+1$ gravity.

The non-linearities of both the equations of motion and the physical configuration/phase spaces, and the presence of gauge symmetries for these theories lead to numerous difficulties in their classical and quantum description. For example, in the path integral quantization of Yang-Mills theory, a gauge-fixing term has to be introduced in the "sum over all configurations"

$$
\int_{\mathcal{A}}[d A] e^{i S_{Y M}}
$$

to ensure that the integration is only taken over one member ${ }^{4} A$ of each gauge equivalence class (see Cheng and $\mathrm{Li}$ (1984) for an illustration of this phenomenon). However, on general grounds we know that a unique and attainable gauge choice does not exist (this is the assertion of the so-called Gribov ambiguity (Gribov 1978, Singer 1978)). In any case, since the expression (2.3) can be made meaningful only in a weak-field approximation where one splits $S_{Y M}=S_{\text {free }}+S_{I}$, with $S_{\text {free }}$ being just quadratic in $A$, this approach has not yielded enough information about other sectors of the theory, where the fields cannot assumed to be weak. The situation is even worse in the perturbative treatment of gravity (where the dynamical variables are given by "small" fluctuations around a fixed Minkowskian background structure), which is known to be non-renormalizable.

Similarly, in the canonical quantization, since no good explicit description of $T^{*}(\mathcal{A} / \mathcal{G})$ available, one quantizes the theory "à la Dirac". This means that one first "quantizes" on the unphysical phase space $T^{*} \mathcal{A}$, "as if there were no constraints". That is, one uses a formal operator representation of the canonical variable pairs $(A, \tilde{E})$, satisfying the canonical commutation relations

$$
\left[\hat{A}_{a}^{i}(x), \hat{\tilde{E}}_{j}^{b}(y)\right]=i \hbar \delta_{j}^{i} \delta_{a}^{b} \delta^{3}(x-y)
$$

the quantum analogues of the Poisson brackets (2.2). These relations are to be thought of as the infinite-dimensional version of the commutation relations $\left[\hat{q}^{i}, \hat{p}_{j}\right]=i \hbar \delta_{j}^{i}$ of the Schrödinger quantization on $\mathbb{R}^{2 n}$ (see Isham (1984) for more details on representation-theoretic considerations of canonical commutation relations). Then a subset of physical wave functions 
$\mathcal{F}_{\text {phy }}=\left\{\Psi_{\text {phy }}(A)\right\}$ is projected out from the space of all wave functions, $\mathcal{F}=\{\Psi(A)\}$, according to

$$
\mathcal{F}_{\text {phy }}^{Y M}=\{\Psi(A) \in \mathcal{F} \mid(\hat{\mathcal{D} E}) \Psi(A)=0\}
$$

i.e., $\mathcal{F}_{\text {phy }}$ consists of all functions that lie in the kernel of the quantized Gauss constraint, $\hat{\mathcal{D E}}$. (For a critical appraisal of the use of such "Dirac conditions", see Loll (1992a).) The notation $\mathcal{F}$ and $\mathcal{F}_{\text {phy }}$ indicates that these are just spaces of complex-valued functions on $\mathcal{A}$ and do not yet carry any Hilbert space structure. Whereas this is not required on the unphysical space $\mathcal{F}$, one does need such a structure on $\mathcal{F}_{\text {phy }}$ in order to make physical statements, for instance, about the spectrum of the quantum Hamiltonian $\hat{H}$. Unfortunately, we lack an appropriate scalar product, i.e. an appropriate gauge-invariant measure $[d A]_{\mathcal{G}}$ in

$$
<\Psi_{\mathrm{phy}}, \Psi_{\mathrm{phy}}^{\prime}>=\int_{\mathcal{A} / \mathcal{G}}[d A]_{\mathcal{G}} \Psi_{\mathrm{phy}}^{*}(A), \Psi_{\mathrm{phy}}^{\prime}(A)
$$

(More precisely, we expect the integral to range over an extension of the space $\mathcal{A} / \mathcal{G}$ which includes also distributional elements (Ashtekar and Isham 1992)). Given such a measure, the space of physical wave functions would be the Hilbert space $\mathcal{H}^{Y M} \subset \mathcal{F}_{\text {phy }}$ of functions square-integrable with respect to inner product (YM5).

Unfortunately, the situation for gravity is not much better. Here the space of physical wave functions is the subspace of $\mathcal{F}$ projected out according to

$$
\mathcal{F}_{\text {phy }}^{G R}=\left\{\Psi(A) \in \mathcal{F} \mid(\hat{\mathcal{D} E}) \Psi(A)=0, \hat{C}_{a} \Psi(A)=0, \hat{C} \Psi(A)=0\right\}
$$

Again, one now needs an inner product on the space of such states. Assuming that the states $\Psi_{\text {phy }}$ of quantum gravity can be described as the set of complex-valued functions on some (still infinite-dimensional) space $\mathcal{M}$, the analogue of (YM5) reads

$$
<\Psi_{\text {phy }}, \Psi_{\text {phy }}^{\prime}>=\int_{\mathcal{M}}[d A] \Psi_{\text {phy }}^{*}(A), \Psi_{\text {phy }}^{\prime}(A),
$$

where the measure $[d A]$ is now both gauge- and diffeomorphism-invariant and projects in an appropriate way to $\mathcal{M}$. If we had such a measure (again, we do not), the Hilbert space 
$\mathcal{H}^{G R}$ of states for quantum gravity would consist of all those elements of $\mathcal{F}_{\text {phy }}^{G R}$ which are square-integrable with respect to that measure. In the - up to now - absence of explicit measures to make (YM5, GR5) well-defined, our confidence in the procedure outlined here stems from the fact that it can be made meaningful for lower-dimensional model systems (such as Yang-Mills theory in $1+1$ and gravity in $2+1$ dimensions), where the analogues of the space $\mathcal{M}$ are finite-dimensional.

Note that there is a very important difference in the role played by the quantum Hamiltonians in gauge theory and gravity. In Yang-Mills theory, one searches for solutions in $\mathcal{H}^{Y M}$ of the eigenvector equation $\hat{H}_{Y M} \Psi_{\text {phy }}(A)=E \Psi_{\text {phy }}(A)$, whereas in gravity the quantum Hamiltonian $\hat{C}$ is one of the constraints and therefore a physical state of quantum gravity must lie in its kernel, $\hat{C} \Psi_{\text {phy }}(A)=0$.

After all that has been said about the difficulties in finding a canonical quantization for gauge theory and general relativity, the reader may wonder whether one can make any statements at all about their quantum theories. The answer for the Yang-Mills theory is of course in the affirmative. Many qualitative and quantitative statements about quantum gauge theory can be derived in a regularized version of the theory, where the flat background space(time) is approximated by a hypercubic lattice. Using fairly refined computational methods, one tries to extract (for either weak or strong coupling) properties of the continuum theory, for example to derive an approximate spectrum of the Hamiltonian $\hat{H}_{Y M}$ in the limit as the lattice spacing tends to zero. Still there are non-perturbative features, the most important being the confinement property of Yang-Mills theory, which also in this framework are not well understood.

Similar attempts to discretize the (Hamiltonian) theory have so far been not very fruitful in the treatment of gravity, because, unlike in Yang-Mills theory, we cannot choose a fixed background metric (with respect to which we could build a hybercubic lattice), without violating the diffeomorphism invariance of the theory. However, there is a more subtle way in which discrete structures do arise in canonical quantum gravity, which will be mentioned in Section 6 below. One important ingredient in these constructions is a set of non-local loop variables on the space $\mathcal{A}$ of connections, which will be the subject of the next section.

\section{Path, loops and holonomies}

In order to appreciate the role of the loop representations which have become such an important ingredient in the canonical quantization of theories on connection space, it is important to get a thorough understanding of the classical properties of the loop variables 
and of how to manipulate them in calculations. This will be done in some detail in this and the following sections. More mathematical properties of loop spaces are discussed in my review article (Loll 1993d).

Given a differentiable, simply connected manifold $\Sigma$ of dimension $d$, a path in $\Sigma$ (Fig.1a) is a continuous map $w$ from a closed interval of the real line $\mathbb{R}$ into $\Sigma$,

$$
\begin{array}{r}
w:\left[s_{1}, s_{2}\right] \rightarrow \Sigma \\
s \mapsto w^{\mu}(s) .
\end{array}
$$

It therefore has the properties of a map between two differentiable manifolds, for example, $\left(C^{r}\right)$ differentiability, piecewise differentiability or non-differentiability, and its tangent vector $\frac{d w}{d s}$ may vanish for some or all parameter values $s$. A loop in $\Sigma$ (Fig.1b) is a closed path, by which we shall mean a continuous map $\gamma$ of the unit interval into $\Sigma$,

$$
\begin{gathered}
\gamma:[0,1] \rightarrow \Sigma \\
s \mapsto \gamma^{\mu}(s), \\
\gamma(0)=\gamma(1) .
\end{gathered}
$$

We will be using such closed paths in the construction of gauge-invariant quantities in pure gauge theory. Open paths play an important role in gauge theory with fermions, where natural gauge-invariant objects are open flux lines with quarks or Higgs fields "glued to the endpoints" (Kogut and Susskind 1975, Gambini et al. 1988, Gambini and Setaro 1993).

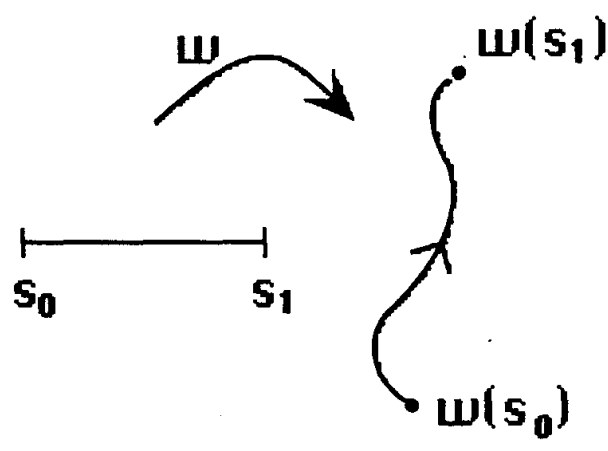

Fig.1a

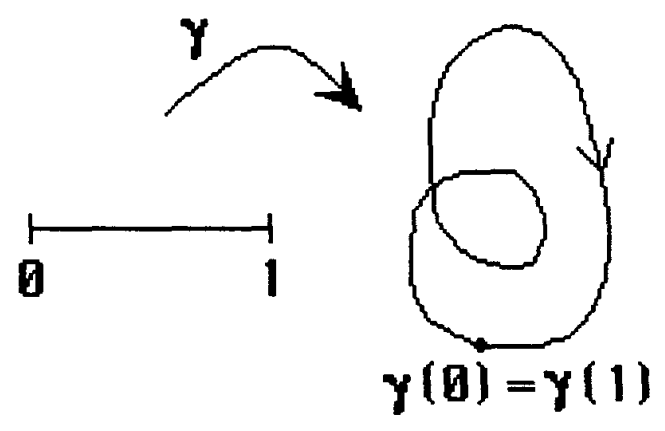

Fig.1b 
The manifold $\Sigma$ may be the real vector space $\mathbb{R}^{d}$, possibly with a Euclidean or Minkowskian metric, but may also be non-linear and topologically non-trivial. In this case the possibility arises of having non-contractible loops, i.e. maps $\gamma$ that cannot be continuously shrunk to a point loop

$$
p_{x}(s)=x \in \Sigma, \quad \forall s \in[0,1]
$$

Note that even if a path is closed it has a distinguished image point, namely, its initial and end point, $\gamma(0)=\gamma(1)$, and that each point in the image of $\gamma$ is labelled by one or more (if there are selfintersections) parameter values $s$.

Suppose now that in an open neighbourhood $V$ of $\Sigma$ we are given a configuration $A \in \mathcal{A}$, the set of all gauge potentials $\Sigma \rightarrow \Lambda^{1} \mathbf{g}$, i.e. a smooth g-valued connection one-form, with $\mathbf{g}$ denoting the Lie algebra of a finite-dimensional Lie group $G$. We have

$$
A(x)=A_{\mu}(x) d x^{\mu}=A_{\mu}^{a}(x) X_{a} d x^{\mu},
$$

where $X_{a}$ are the algebra generators in the fundamental representation of $\mathbf{g}$ ( $a=1 \ldots \operatorname{dim}$ $G)$ and $x^{\mu}, \mu=1 \ldots d$, a set of local coordinates on $V$.

The holonomy $U_{w}$ of a path $w^{\mu}(s)$ with initial point $s_{0}$ and endpoint $s_{1}$ (whose image is completely contained in $V$ ) is the solution of the system of differential equations

$$
\frac{d U_{w}\left(s, s_{0}\right)}{d s}=A_{\mu}(x) \frac{d w^{\mu}}{d s} U_{w}\left(s, s_{0}\right), \quad s_{0} \leq s \leq s_{1},
$$

with $x=w(s)$, subject to the initial condition

$$
U_{w}\left(s_{0}, s_{0}\right)=e
$$

where $e$ denotes the unit element in $G$. Note that this definition only makes sense for at least piecewise differentiable paths $w$. The solution of (3.5) is given by the path-ordered exponential of $A$ along $w$, 


$$
\begin{aligned}
& U_{w}\left(s_{1}, s_{0}\right)=\mathrm{P} \exp \int_{s_{0}}^{s_{1}} A_{\mu}(w(t)) \dot{w}^{\mu}(t) d t:= \\
& :=\mathbb{1}+\sum_{n=1}^{\infty} \int_{s_{0}}^{s_{1}} d t_{1} \int_{t_{1}}^{s_{1}} d t_{2} \ldots \int_{t_{n-1}}^{s_{1}} d t_{n} A_{\mu_{n}}\left(w\left(t_{n}\right)\right) \ldots A_{\mu_{1}}\left(w\left(t_{1}\right)\right) \dot{w}^{\mu_{n}}\left(t_{n}\right) \ldots \dot{w}^{\mu_{1}}\left(t_{1}\right) .
\end{aligned}
$$

Note that (3.7) implies the composition law $U_{w}\left(s_{2}, s_{0}\right)=U_{w}\left(s_{2}, s_{1}\right) U_{w}\left(s_{1}, s_{0}\right)$, for $s_{0} \leq s_{1} \leq$ $s_{2}$. An alternative definition for $U_{w}$ that does not need differentiability of the path and employs an approximation of $w$ by $n$ straight line segments $\left(x_{i}-x_{i-1}\right)$ is as the limit

$U_{w}\left(s_{1}, s_{0}\right)=\lim _{n \rightarrow \infty}\left(1+A\left(x_{n}\right)\left(x_{n}-x_{n-1}\right)\right)\left(1+A\left(x_{n-1}\right)\left(x_{n-1}-x_{n-2}\right)\right) \ldots\left(1+A\left(x_{1}\right)\left(x_{1}-x_{0}\right)\right)$,

with sup $\left\|x_{i}-x_{i-1}\right\| \rightarrow 0$ as $n$ increases (Corrigan and Hasslacher 1979), and where $x_{0}=$ $w\left(s_{0}\right), x_{1}, \ldots, x_{n}=w\left(s_{1}\right)$ is a set of $n+1$ points ordered along the path $w$ (Fig.2).

Fig.2

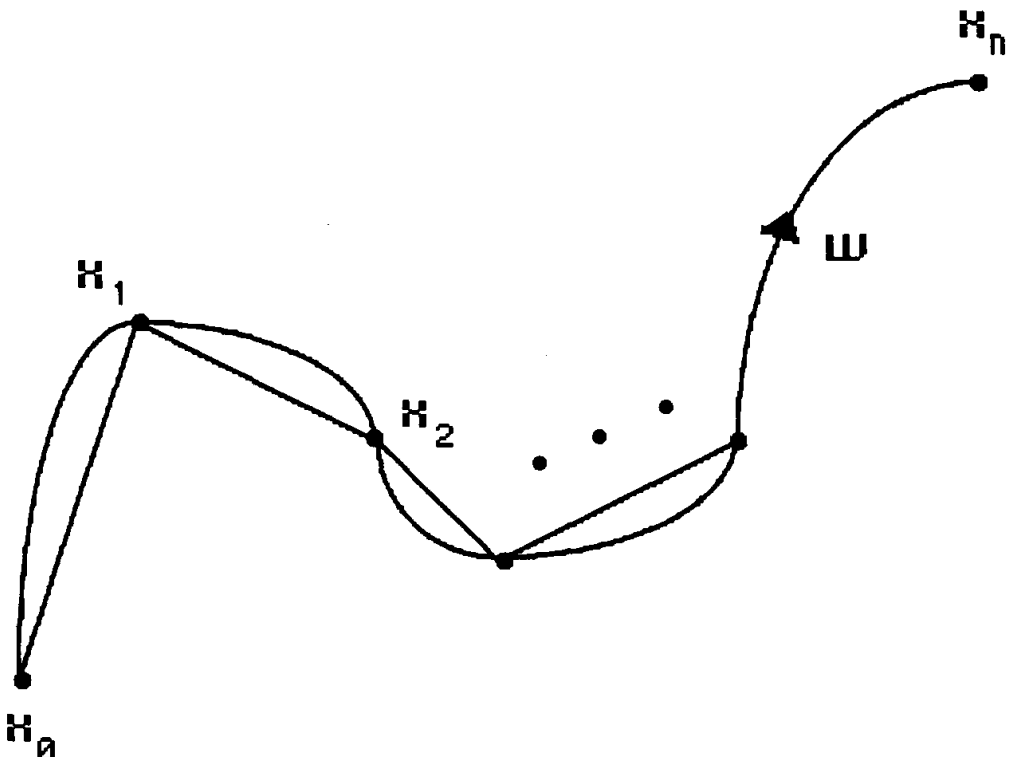

The holonomy $U_{w}$ takes its values in $G$ and transforms under gauge transformations (smooth functions $g: V \rightarrow G$ ) according to

$$
U_{w}\left(s_{1}, s_{0}\right) \stackrel{g}{\longmapsto} g^{-1}\left(w\left(s_{1}\right)\right) U_{w}\left(s_{1}, s_{0}\right) g\left(w\left(s_{0}\right)\right)
$$


Note this would not be true if we had allowed for discontinuities of the path $w$. The corresponding change of the gauge potential is straightforwardly computed from equation (3.5),

$$
A_{\mu}(x) \stackrel{g}{\longrightarrow} g^{-1}(x) A_{\mu}(x) g(x)-\frac{d g^{-1}(x)}{d x^{\mu}} g(x) \equiv g^{-1}(x) A_{\mu}(x) g(x)+g^{-1}(x) \frac{d g(x)}{d x^{\mu}}
$$

Another property of $U_{w}\left(s_{1}, s_{0}\right)$ following from (3.5) is its invariance under smooth orientationpreserving reparametrizations $f$ of $w$, i.e.

$$
w(s)=w^{\prime}(f(s)) \Longrightarrow U_{w}\left(s_{1}, s_{0}\right)=U_{w^{\prime}}\left(f\left(s_{1}\right), f\left(s_{0}\right)\right),
$$

where $\frac{d f}{d s}>0, \forall s$. The term "non-integrable" (i.e. path-dependent) "phase factor" for $U_{w}$ was introduced by Yang (1974), in generalization of its abelian version for $U(1)$-electromagnetism. It is a well-known result in mathematics that the connection $A$ can (up to gauge transformations) be reconstructed from the knowledge of the holonomies of the closed curves based at a point $x_{0} \in \Sigma$ (see Kobayashi and Nomizu (1969), Lichnerowicz (1976) for details on the concept of holonomy group and related issues). The reader may consult the paper by Barrett (1991) for an account of the mathematical development of these so-called reconstruction theorems, and for more references. The details of such theorems depend on the mathematical setting, for instance whether the underlying fibre bundle is differentiable or just topological. Also generalizations of the concept of connection are possible.

The above description is valid only in a local neighbourhood $V$ of $\Sigma$. The appropriate global description is afforded by the mathematical theory of principal fibre bundles $P: G \rightarrow$ $P \rightarrow \Sigma$ over the base manifold $\Sigma$ with typical fibre $G$, with $A^{P}$ a connection one-form on $P$. Typically $P$ has no global cross sections (this depends both on the group $G$ and the manifold $\Sigma$ ), and then $A^{P}$ can be identified with the one-form (3.4) only in a coordinate patch $V \subset \Sigma$, using a local cross section. The global implications of this fact are well known and will not be addressed here. The holonomy variables are global in the sense that because of their gaugeinvariance they do not "see" non-trivial transition functions between coordinate patches on the manifold $\Sigma$. In the (for the present discussion) important cases of $G=S U(2), S L(2, \mathbb{C})$ and any three-dimensional $\Sigma$, global cross sections do exist however and hence there is no need for "patching" on the base manifold. Note that for $F=0$ (the case of a "flat connection"), where $F$ is the field strength tensor (2.1), $U_{w}$ depends only on the homotopy of the path $w$.

Let us now concentrate on the special case when $w$ is a closed path $\gamma$ in $\Sigma$, with associated holonomy, 


$$
U_{\gamma}(A)=\mathrm{P} \exp \oint_{\gamma} A_{\mu}(x) d x^{\mu}
$$

For a loop $\gamma$ based at a point $x_{0} \in \Sigma$ there is a natural way of constructing a gaugeinvariant quantity, namely,

$$
h[\gamma, A]:=\frac{1}{N} \operatorname{tr} U_{\gamma}=\frac{1}{N} \operatorname{tr} P \exp \oint_{\gamma} A_{\mu}(x) d x^{\mu},
$$

the traced holonomy or so-called Wilson loop (introduced by Wilson (1974) as an indicator of confining behaviour in lattice gauge theory). The trace is taken in a linear representation $R$ of the group $G$, and the normalization factor in front depends on the dimension $N$ of $R$. Under a gauge transformation, $U_{\gamma, x_{0}}$ transforms homogeneously according to (3.8), but the matrices $g\left(x_{0}\right)$ and $g^{-1}\left(x_{0}\right)$ cancel each other upon taking the trace. Because of the cyclicity of the trace, $h[\gamma, A]$ is independent of the choice of base point $x_{0}=\gamma(0)=\gamma(1)$ of $\gamma$. Note that $h[\gamma, A]$ is invariant under orientation-preserving reparametrizations of $\gamma$ and is therefore a function on unparametrized loops, i.e. reparametrization equivalence classes $\bar{\gamma}$, where any two members of $\bar{\gamma}$ are related by a smooth orientation-preserving bijection $b: S^{\mathbf{1}} \mapsto S^{\mathbf{1}}$, $\frac{d b}{d s}>0$. It follows that the Wilson loop is a function on the cartesian product of the quotient spaces, $h[\gamma, A] \in \mathcal{F}\left(\mathcal{A} / \mathcal{G} \times \Omega \Sigma / \operatorname{Diff}^{+}\left(S^{1}\right)\right)$.

The holonomy of small closed loops measures the curvature (or field strength) in internal space. For a small square loop $\gamma$ of side length $\epsilon$ in a coordinate chart around the point $x \in \Sigma$, the base point of $\gamma$, the holonomy $U_{\gamma}$ can be expanded as

$$
U_{\gamma}=\mathbb{1}+F_{\mu \nu}^{a}(x) X_{a} \epsilon^{2}+O\left(\epsilon^{3}\right),
$$

where $\gamma$ is defined by its four corners, $\left(x, x+\epsilon \vec{e}_{\mu}, x+\epsilon \vec{e}_{\mu}+\epsilon \vec{e}_{\nu}, x+\epsilon \vec{e}_{\nu}\right)$, with $\vec{e}_{\mu}$ denoting the unit vector in $\mu$-direction. Note that in the non-abelian gauge theory, $F_{\mu \nu}(x)=F_{\mu \nu}^{a}(x) X_{a}$ is not an observable, since it transforms non-trivially under gauge transformations. A geometric description of the parallel transport associated with the Ashtekar connection in gravity is given in the review paper by Kuchaŕ (1993).

Performing a similar expansion for the traced holonomy of the infinitesimal loop $\gamma$, we obtain 


$$
h[\gamma, A]=\operatorname{tr} U_{\gamma}=1+\frac{1}{N} \sum_{a} F_{\mu \nu}^{a}(x) \operatorname{tr} X_{a} \epsilon^{2}+\frac{1}{N} \sum_{a, b} F_{\mu \nu}^{a}(x) F_{\mu \nu}^{b}(x) \operatorname{tr} X_{a} X_{b} \epsilon^{4}+O\left(\epsilon^{5}\right)
$$

(no sum over $\mu, \nu$ ). For a semi-simple Lie algebra $\mathbf{g}$ we can always find a basis of generators $X_{a}$ such that $\operatorname{tr} X_{a} X_{b}=\delta_{a b}$. Moreover, for $G=S U(N)$ (more generally, for any subgroup of the special linear group $S L(N, \mathbb{C})$, we have tr $X_{a}=0$, and the expansion therefore reduces to

$$
h[\gamma, A]=1+\frac{1}{N} \sum_{a} F_{\mu \nu}^{a}(x) F_{\mu \nu}^{a}(x) \epsilon^{4}+O\left(\epsilon^{5}\right)
$$

These expansions illustrate the way in which local gauge-invariant information about the curvature or field strength $F$ is contained in the Wilson loop $h[\gamma, A]$. In concrete calculations involving an unparametrized loop $\vec{\gamma}$ one usually works with a chosen member $\gamma$ from the equivalence class $\bar{\gamma}$ and then ensures the end result is independent of this choice. There have also been attempts to set up a loop calculus that is intrinsically reparametrization-invariant (Gambini and Trias 1981, 1983, Migdal 1983, Gambini 1991, Tavares 1993).

In gravity, the Wilson loops are not observables, since they are not invariant under the full set of gauge transformations of the theory. However, in this context there is yet another reason to consider variables that depend on closed curves in $\Sigma$, since observables in gravity also have to be invariant under diffeomorphisms of $\Sigma$. This means going one step further in the construction of such observables, by considering Wilson loops that are constant along the orbits of the $\operatorname{Diff}(\Sigma)$-action,

$$
h[\varphi \circ \gamma, A]=h[\gamma, A], \forall \varphi \in \operatorname{Diff}(\Sigma)
$$

Such a loop variable will just depend on what is called the generalized knot class $K(\gamma)$ of $\gamma$ ("generalized" since $\gamma$ may possess self-intersections, which is not usually considered in knot theory). Note that a similar construction for taking care of diffeomorphism invariance is not very contentful if we start from a local scalar function, $\phi(x)$, say. The analogue of (3.17) would then tell us that $\phi$ had to be constant on any connected component of $\Sigma$. The set of such variables is not big enough to account for all the degrees of freedom of gravity. In contrast, loops can carry diffeomorphism-invariant information such as their number of self-intersections, number of points of non-differentiability, etc. Although no complete classification of (generalized) knots is known at this point, they are quantities that are easier 
to visualize and manipulate than their counterparts in the metric formulation of gravity, the equivalence classes Riem $(\Sigma) / \operatorname{Diff}_{0}(\Sigma)$ of Riemannian metrics modulo diffeomorphisms. The relation between quantum gravity and knot theory is the subject of Brügmann's contribution in this volume.

The use of non-local holonomy variables in gauge field theory was pioneered by Mandelstam in his 1962 paper on "Quantum electrodynamics without potentials" (Mandelstam 1962), and later generalized to the non-abelian theory by Bialynicki-Birula (1963) and himself (Mandelstam 1968). Another upsurge of interest in path-dependent formulations of YangMills theory took place at the end of the seventies, following the observation of the close formal resemblance of a particular form of the Yang-Mills equations in terms of holonomy variables and the equations of motion of the two-dimensional non-linear sigma model (Aref'eva 1979, Polyakov 1979). Another set of equations for the Wilson loop were derived by Makeenko and Migdal $(1979,1981)$. The hope underlying such approaches has always been that one may be able to find a set of basic variables for Yang-Mills theory, which are better suited to its quantization than the local gauge potentials $A(x)$. Due to their simple behaviour under local gauge transformations, the holonomy or the Wilson loop seem like ideal candidates. Hence the aim has been to derive suitable differential equations in loop space for the holonomy $U_{\gamma}$, its trace, $h[\gamma]$, or for their vacuum expectation values, which are to be thought of as the analogues of the usual local Yang-Mills equations of motion. The fact that none of these attempts have been very successful can be attributed to a number of reasons.

(a) To make sense of differential equations on loop space, one first of all has to introduce a suitable topology, and then set up a differential calculus on $\Omega \Sigma$. Even if we give $\Omega \Sigma$ locally the structure of a topological vector space, and are able to define differentiation, this in general will ensure neither the existence of inverse and implicit function theorems nor theorems on the existence and uniqueness of solutions of differential equations. Such issues have only received scant attention in the past.

(b) Since the space of all loops in $\Sigma$ is so much larger than the set of all points in $\Sigma$, one expects that not all loop variables will be independent. This expectation is indeed correct, as will be explained in the next section. Still, the ensuing redundancy is hard to control in the continuum theory, and has therefore obscured many attempts of establishing an equivalence between the connection and the loop formulation of gauge theory. For example, there is no action principle in terms of loop variables, which leaves considerable freedom of how to derive "loop equations of motion". As another consequence, perturbation theory in the loop approach always had to fall back on the perturbative expansion in terms of the connection variable $A$. 


\section{Identities satisfied by holonomies and their traces}

Central to the loop description of gauge theory and gravity are certain identities satisfied by the holonomies and Wilson loops, which follow from their definitions (3.7) and (3.13). They have to be imposed as constraints if we re-interpret those theories as theories on some loop space, without reference to the space $\mathcal{A}$ of connections. Take $G$ to be the gauge group $G L(N, \mathbb{C})$ or one of its subgroups $(U(N), S U(N), S O(N)$, etc.) in its fundamental representation in terms of $N \times N$ (complex) matrices. For two loops $\gamma_{1}$ and $\gamma_{2}$ based at $x$, we have

$$
U_{\gamma_{1} \circ \gamma_{2}}(A)=U_{\gamma_{2}}(A) \cdot U_{\gamma_{1}}(A), \quad \forall A,
$$

with $\circ$ denoting the loop composition, $\cdot$ matrix multiplication and $U_{\gamma}$ defined by (3.12). For the inverse loop $\gamma^{-1}$ of $\gamma, \gamma^{-1}(s):=\gamma(1-s)$, we have

$$
U_{\gamma^{-1}}=\left(U_{\gamma}\right)^{-1}
$$

Here and in the rest of this article, dependence of $U_{\gamma}(A)$ and $h[\gamma, A]$ on $A$ will not be written explicitly, and we will also use the customary notation $T(\gamma):=h[\gamma, \cdot]$ for the Wilson loops. Since (4.2) holds also for open paths $\gamma$, we derive the important retracing identity

$$
U_{\gamma}=U_{\gamma^{\prime}}, \quad \text { for } \gamma^{\prime}=\left(\left(\gamma_{1} \circ w\right) \circ w^{-1}\right) \circ \gamma_{2}
$$

where $\gamma_{1} \circ \gamma_{2}=\gamma, w$ is an open path "glued to $\gamma$ " (see Fig.3).

Fig.3

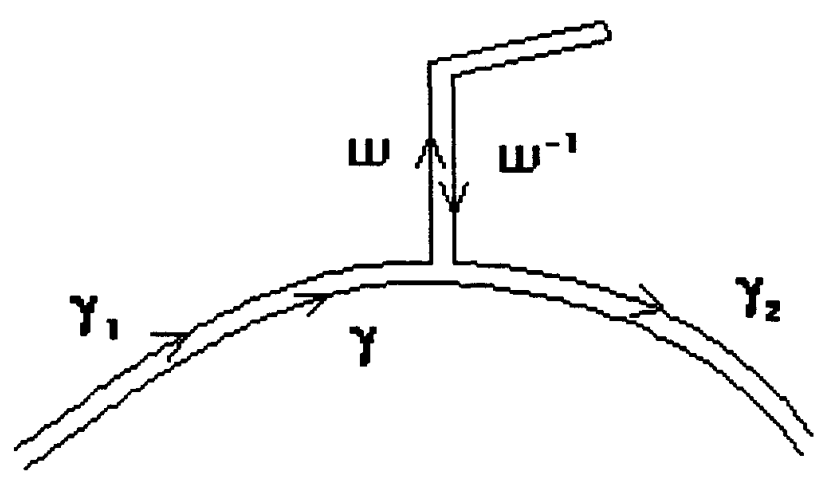


Although the composition of loops is non-associative, it is easily seen that $U_{\gamma^{\prime}}$ in (4.3) is independent of the order of composition of the loop segments; for example, we could have chosen $\gamma^{\prime}=\gamma_{1} \circ\left(w \circ\left(w^{-1} \circ \gamma_{2}\right)\right)$ instead. The property (4.3) motivates the introduction of an equivalence relation on paths. A loop $\gamma$ is called a thin loop if it is homotopic to the trivial point loop $p$ by a homotopy of loops whose image lies entirely within the image of $\gamma$. Two loops (paths) $\gamma_{1}$ and $\gamma_{2}$ are called thinly equivalent if $\gamma_{1} \circ \gamma_{2}^{-1}$ is a thin loop. Note that two loops that differ by an orientation-preserving reparametrization are always thinly equivalent.

We will denote the equivalence class of a loop $\gamma$ by $\bar{\gamma}$, and the set of all loops in $\Sigma$ based at $x_{0}$ by $\Omega_{x_{0}} \Sigma$. Under the above equivalence relation, the set of equivalence classes $\Omega_{x_{0}} \Sigma / \sim$ acquires a group structure, which is characterized by the relations

$$
\begin{aligned}
& \bar{\beta}=\bar{\gamma} \quad \text { if } \beta=\gamma \bmod \text { retracing/reparametrization } \\
& \bar{\gamma} \circ \bar{\gamma}^{-1}=\bar{p}_{x_{0}} \\
& (\bar{\alpha} \circ \bar{\beta}) \circ \bar{\gamma}=\bar{\alpha} \circ(\bar{\beta} \circ \bar{\gamma}) \quad \text { (associativity) }
\end{aligned}
$$

with the unit $\bar{p}_{x_{0}}$, the equivalence class of the constant loop (3.3). This group is usually called the "loop group" or the "group of loops" (not to be confused with the loop groups of maps from $S^{1}$ into a Lie group $G$ à la Pressley and Segal (1986)), and will be denoted by $\mathcal{L} \Sigma$. The group $\mathcal{L} \Sigma$ is non-abelian and, as shown by Durhuus and Leinaas (1982), not locally compact, which means it is "very large" even locally. This feature leads to complications when one tries to define a Fourier transform on $\mathcal{L} \Sigma$. The loop group has been discussed by many authors in view of its application to physics (Mensky 1978, Fröhlich 1979, Gambini and Trias 1981, Durhuus and Leinaas 1982, Anandan 1983, Fischer 1986, Barrett 1991, Ashtekar and Isham 1992). Its construction has been around in the mathematics literature for a long time (see the remarks and references in Barrett (1991)). Some toy examples of simpler loop groups in two dimensions are contained in Durhuus and Leinaas (1982). A simplified version for polygonal loops on $\mathbb{R}^{4}$ is discussed by Gambini and Trias (1981). - Some authors prefer to induce an equivalence relation on loops by defining $\beta \sim \gamma$ if $U_{\beta}(A)=U_{\gamma}(A), \forall A$, which explicitly refers to the space of connections (Lewandowski 1993, Ashtekar and Lewandowski 1993b).

Note that by virtue of (4.1), the holonomy mapping $U$ may be viewed as a group homomorphism $U: \mathcal{L} \Sigma \rightarrow G$. The Wilson loops (3.13) may therefore be regarded as the characters of a representation of the loop group $\mathcal{L} \Sigma$ (Fröhlich 1979, Durhuus and Leinaas 1982, Anandan 1983). This viewpoint may be profitably exploited in some questions arising in the quantum theory.

There are analogous identities satisfied by the traced holonomies, characterizing them as 
a particular subset of complex-valued functions on $\Omega \Sigma \times \mathcal{A} / \mathcal{G}$. Independently of the gauge group $G$, we have the identities

$$
T\left(\gamma_{1} \circ \gamma_{2}\right)=T\left(\gamma_{2} \circ \gamma_{1}\right)
$$

because of the cyclicity of the trace, and again a retracing identity,

$$
T(\gamma)=T\left(\gamma^{\prime}\right), \quad \gamma, \gamma^{\prime} \text { related as in }(4.3)
$$

Another set of identities are the so-called Mandelstam constraints, whose form depends on the dimension $N$ of the group matrices. They can be systematically derived from the identity of $N$-dimensional $\delta$-functions,

$$
\sum_{\pi \in \mathcal{S}_{N+1}}(-1)^{\sigma(\pi)} \delta_{i_{1}, \pi\left(j_{1}\right)} \ldots \delta_{i_{N+1}, \pi\left(j_{N+1}\right)}=0, \quad i_{k}, j_{k}=1 \ldots N
$$

with the sum running over all permutations $\pi$ of the symmetric group of order $N+1, \mathcal{S}_{N+1}$, and $\sigma(\pi)$ denoting the parity of the permutation. Contracting $N+1$ holonomy matrices $U_{\gamma}$ with (4.7) results in a trace identity for (combinations of) $N+1$ loops. For $N=1$, we have

$$
T(\alpha) T(\beta)-T(\alpha \circ \beta)=0
$$

and for $N=2$,

$$
T(\alpha) T(\beta) T(\gamma)-\frac{1}{2}(T(\alpha \beta) T(\gamma)+T(\beta \gamma) T(\alpha)+T(\alpha \gamma) T(\beta))+\frac{1}{4}(T(\alpha \beta \gamma)+T(\alpha \gamma \beta))=0
$$

Note that the Mandelstam identities are non-linear algebraic equations on the functions $T$. If we want to consider traced holonomies of specific subgroups of $G L(N, \mathbb{C})$, there will be more identities satisfied by $T$, for example, deriving from a condition det $U_{\gamma}=1$ (see Gliozzi and Virasoro (1980), Gambini and Trias (1986) and the next section for some selected cases). 
We may use the functions $T$ to induce an equivalence relation on the loop space $\Omega \Sigma$ by defining

$$
\beta \sim \gamma \quad \text { if } \quad T(\beta)=T(\gamma), \forall A .
$$

The composition law for equivalence classes $\bar{\gamma}$,

$$
\bar{\gamma}_{1} \circ \bar{\gamma}_{2}:=\overline{\gamma_{1} \circ \gamma_{2}}
$$

induces an abelian group structure on $\Omega \Sigma / \sim$ if in addition the relation $T(\alpha)=T(\beta) \Rightarrow$ $T(\alpha \circ \gamma)=T(\beta \circ \gamma)$ is satisfied for all $\gamma$ (Gambini and Trias 1986, Ashtekar and Isham 1992).

\section{Equivalence between gauge potentials and holonomies}

The importance of the (traced) holonomies lies in the fact that from them one can reconstruct gauge-invariant information about the gauge potential $A$. The content of the so-called reconstruction theorems is to specify a set of algebraic and differential conditions on a set of functions on loop space which ensure that from them one can uniquely compute the corresponding equivalence class $[A] \in \mathcal{A} / \mathcal{G}$.

The best-known case is that of holonomies based at a point, for which various versions of the reconstruction theorem are available, depending on the mathematical setting. Although known to mathematicians for a long time, they have been regularly rediscovered by physicists. A very detailed discussion and derivation is contained in the paper by Barrett (1991). I quote here his reconstruction theorem for differentiable principal fibre bundles (related treatments may be found in Fröhlich (1979), Gliozzi and Virasoro (1980), Giles (1981), Anandan (1983)):

Reconstruction Theorem. Suppose $\Sigma$ is a connected manifold with basepoint $x_{0}$ and the map $H: \Omega_{x_{0}} \Sigma \rightarrow G$ satisfies the following conditions:

(i) $H$ is a homomorphism of the composition law of loops, $H\left(\gamma_{1} \circ \gamma_{2}\right)=H\left(\gamma_{2}\right) H\left(\gamma_{1}\right)$.

(ii) $H$ takes the same values on thinly equivalent loops: $\gamma_{1} \sim \gamma_{2}$ if $\gamma_{1} \circ \gamma_{2}^{-1}$ is thin (cf. Sec.4).

(iii) For any smooth finite-dimensional family of loops $\tilde{\psi}: U \rightarrow \Omega_{x_{0}} \Sigma$, the composite map $H \psi: U \rightarrow \Omega_{x_{0}} \Sigma \rightarrow G$ is smooth. 
Then there exists a differentiable principal fibre bundle $P(\Sigma, G, \pi)$, a point $p \in \pi^{-1}\left(x_{0}\right)$ and a connection $\Gamma$ on $P$ such that $H$ is the holonomy mapping of $\left(P, \Gamma, x_{0}\right)$. (In (iii) above, $U$ is an open subset of $\mathbb{R}^{n}$ (parametrizing the family), for any $n$, and $\tilde{\psi}$ is smooth in the sense that the associated map $\psi: U \times I \rightarrow \Sigma$ is continuous and piecewise $C^{\infty}$ with respect to the the loop parameter $t \in I$.)

Note that in this formulation the principal bundle $P$ is not fixed a priori, but only the base space $\Sigma$ and the fibre $G$ are. Fixing $P$ would amount to fixing the homotopy class of the holonomy mapping.

Let us go back to the discussion of the reconstruction theorem, now for the case of the traced holonomies. Since we already have a reconstruction theorem for the holonomies, it suffices to show one can recover the holonomies from the Wilson loops. The main task is to find algebraic conditions analogous to (i) and (ii) above, characterizing uniquely the traced holonomies as a subset of complex-valued functions on $\Omega \Sigma$. It seems to be much harder to come up with a set of necessary and sufficient conditions, moreover, these conditions now depend on the gauge group $G$. To my knowledge, the problem of giving a complete set of such conditions for a general gauge group $G$ has not been solved. The most advanced results in this context are those obtained by Giles (1981).

The content of his reconstruction theorem is essentially as follows: Given any complexvalued function $F(\gamma)$ on the loop space $\Omega \Sigma$ satisfying the Mandelstam identities of order $N$ (and possibly some additional identities, characterizing a specific subgroup of $G L(N, \mathbb{C})$ ), retracing and reparametrization invariance, equation (4.8) and appropriate smoothness conditions, one can construct (modulo a residual gauge freedom) $N \times N$ matrices $U_{\gamma} \in G L(N, \mathbb{C})$ (or of the subgroup in question) such that the traces of products $U_{\gamma_{j}} \cdot U_{\gamma_{i}} \cdot \ldots$ are exactly given by $F\left(\ldots \circ \gamma_{i} \circ \gamma_{j}\right)$.

Giles gives an explicit way of reconstructing the holonomies from the traced holonomies, which is very useful in practical applications. However, his results are incomplete in at least two aspects. Firstly, in addition to the usual Mandelstam constraints, there are inequalities restricting the range of the Wilson loops, i.e. given a set of $n$ Wilson loops, they can in general not attain arbitrary complex values, even if all the Mandelstam identities are fulfilled (Loll 1993b). Secondly, it has not been proven that by running through all admissible $F(\gamma)$ 's one recovers indeed all possible holonomy configurations $U_{\gamma}$. In general the $U_{\gamma}$ 's so obtained will form a subgroup of $G$. This however can only happen if $G$ is non-compact, a well-studied example being that of $G=S L(2, \mathbb{C})$ (Goldberg et al. 1992).

For the sake of illustration, and because of their importance in many applications, here 
are the explicit Mandelstam constraints for $G=S L(2, \mathbf{C})$, in the fundamental representation by complex $2 \times 2$-matrices. One finds

(a) $\quad T($ point loop $p)=1$

(b) $\quad T\left(\gamma_{1}\right)=T\left(\gamma_{2}\right), \quad$ if $\gamma_{1}$ and $\gamma_{2}$ are thinly equivalent

(c) $\quad T\left(\gamma_{1} \circ \gamma_{2}\right)=T\left(\gamma_{2} \circ \gamma_{1}\right)$

(d) $\quad T(\gamma)=T\left(\gamma^{-1}\right)$

(e) $\quad T\left(\gamma_{1}\right) T\left(\gamma_{2}\right)=\frac{1}{2}\left(T\left(\gamma_{1} \circ \lambda \circ \gamma_{2} \circ \lambda^{-1}\right)+T\left(\gamma_{1} \circ \lambda \circ \gamma_{2}^{-1} \circ \lambda^{-1}\right)\right)$.

In (e), $\lambda$ is a path connecting a point on $\gamma_{1}$ with a point on $\gamma_{2}$, as illustrated by Fig.4. Note that (e) implies both (a) and (d). However, the conditions have been separated in this way since (a)-(c) hold for any group, whereas (d) and (e) are true just for $S L(2, \mathbf{C})$. Presumably the set (9.1) exhaust all the Mandelstam constraints for this particular group and representation, although I am not aware of the existence of a formal proof. In any case, it can be shown that there are holonomies $U_{\gamma}$ that cannot be reconstructed from the Wilson loops, namely those that lie in the subgroup of so-called null rotations. However, in a sense this incompleteness is negligible in the physical applications considered so far (Goldberg et al. 1992), and in fact the Wilson loops are "as complete as they could be" (Ashtekar and Lewandowski 1993a).

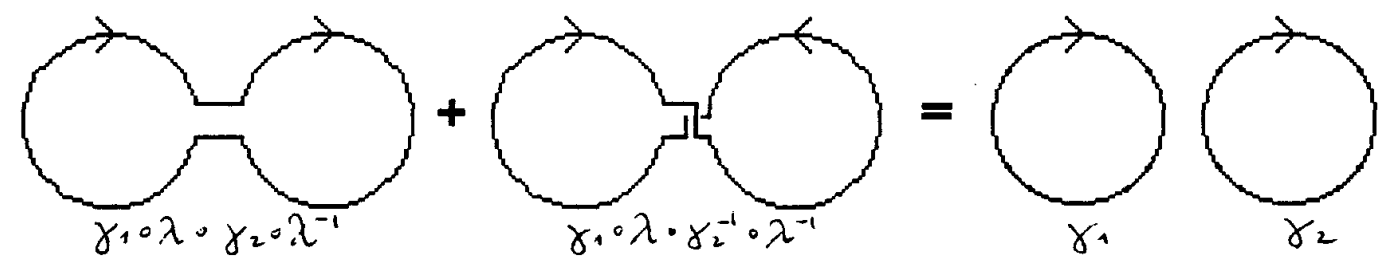

Fig.4

If one wants to restrict the group $G$ to be described by the Wilson loops to a subgroup of $S L(2, \mathbb{C})$, there are further conditions that have to be imposed on the loop variables, including inequalities. For the subgroup $S U(2) \subset S L(2, \mathbb{C})$, one has 
(a) $\quad T(\gamma)$ real

(b) $\quad-1 \leq T(\gamma) \leq 1$

(c) $\quad\left(T\left(\gamma_{1} \circ \gamma_{2}\right)-T\left(\gamma_{1} \circ \gamma_{2}^{-1}\right)\right)^{2} \leq 4\left(1-T\left(\gamma_{1}\right)^{2}\right)\left(1-T\left(\gamma_{2}\right)^{2}\right)$

whereas the analogous conditions for the subgroup $S U(1,1) \subset S L(2, \mathbb{C})$ are

(a) $\quad T(\gamma)$ real

(b) $\quad T\left(\gamma_{1}\right)^{2} \leq 1$ and $T\left(\gamma_{2}\right)^{2} \leq 1 \Rightarrow$

$$
\left(T\left(\gamma_{1} \circ \gamma_{2}\right)-T\left(\gamma_{1} \circ \gamma_{2}^{-1}\right)\right)^{2} \geq 4\left(1-T\left(\gamma_{1}\right)^{2}\right)\left(1-T\left(\gamma_{2}\right)^{2}\right)
$$

As indicated by the dots, there are other inequalities for more complicated loop configurations, involving more than two loops $\gamma_{i}$. Note that all of the above relations hold independently of $A$, and hence may be interpreted as constraints on loop space functions on $\Omega \Sigma$, rather than as identities on the loop-dependent functions on the space of connections, i.e. as functions on $\Omega \Sigma \times \mathcal{A} / \mathcal{G}$. Also at least some of these algebraic conditions can be interpreted as conditions on the underlying loop space. For example, (5.1d) may be interpreted as a condition that $T$ is a function of unoriented loops.

If one wants to reformulate a theory purely in terms of loop variables, without making any reference to the connection variables $A$, one has to come up with a complete set of conditions of the type discussed above to ensure equivalence with the usual local formulation. This is a non-trivial task, and not always appreciated in physical applications. The difficulty of proving rigorous reconstruction theorems for the Wilson loops has somewhat hampered this kind of "pure loop approach". The most progress in isolating the true degrees of freedom in such an approach has been made on the hypercubic lattice (Loll 1992b,1993a,c). - Once a reconstruction theorem has been proven, the method is potentially very powerful, since any gauge-invariant statement in terms of the connection $A$ is in principle expressible in terms of the traced holonomies $T(\gamma)$.

Alternatively, one may follow a less radical approach which still incorporates the gauge potentials. For example, one may define a ( $G$-dependent) equivalence relation on loops by identifying two loops if their associated Wilson loops agree for all possible configurations $A(x)$ (4.12). Equivalence classes of this kind are sometimes called "hoops". The quotient 
space of loop space one obtains in this way is of course exactly the one one would like to use as a domain space when constructing a formulation exclusively in terms of loop variables. Another possibility is to construct a "loop transform" in the quantum theory which allows one to translate wave functions and operators from the connection to the loop representation. If it can be shown to exist rigorously for particular spaces of connections, this transform automatically takes care of algebraic constraints among the Wilson loop operators, i.e. quantum analogues of the Mandelstam constraints.

Incidentally, there have also been attempts to reformulate gauge theories in terms of the field strengths $F$ instead of the gauge potentials $A$, because they have a simpler, homogeneous transformation behaviour. However, it is well known that gauge-inequivalent connection configurations may have the same field strength (although this does not happen for "generic" connections), and hence a rigorous reconstruction theorem does not exist. A discussion of this degeneracy and a guide to the literature is contained in the paper by Mostow and Shnider (1983).

Finally, let me emphasize that the equivalence of the connection and the loop description at the classical level does not necessarily mean that quantization with the Wilson loops as a set of basic variables will be equivalent to a quantization in which the connections $A$ are turned into fundamental quantum operators. One may indeed hope that at some level, those two possibilities lead to different results, with the loop representation being better suited for the description of the non-perturbative structure of the theory. 


\section{Some lattice results on loops}

The contents of this section concerns the gauge-theoretic application of taking the Wilson loops as a set of basic variables, although some of the techniques and conclusions may be relevant for gravitational theories too. The lattice discretization is particularly suited to the loop formulation, because the gauge fields are taken to be concentrated on the onedimensional links $l_{i}$ of the lattice. The Wilson loops are easily formed by multiplying together the holonomies $U_{l_{1}} \ldots U_{l_{n}}$ of a set of contiguous, oriented links that form a closed chain on the lattice (see Fig.5),

$$
T(\gamma)=\operatorname{Tr}_{R} U_{l_{1}} U_{l_{2}} \ldots U_{l_{n}}
$$

We take the lattice to be the hypercubic lattice $N^{d}$, with periodic boundary conditions $(N$ is the number of links in each direction), and the gauge group $G$ to be $S U(2)$, again in the fundamental representation. Although the lattice is finite, there are an infinite number of closed contours one can form on it, and hence an infinite number of Wilson loops. At the same time, the number of Mandelstam constraints (5.1) is infinite. The only constraints that cause problems are those of type (e), because of their non-linear and highly coupled character. Nevertheless, as I showed in Loll (1992b), it is possible to solve this coupled set of equations and obtain an explicit local description of the finite-dimensional physical configuration space,

$$
\mathcal{C}_{\text {phy }}=\left.\frac{\mathcal{A}}{\mathcal{G}}\right|_{\text {lattice }}=\frac{\{T(\gamma), \gamma \text { a lattice loop }\}}{\text { Mandelstam constraints }} .
$$

in terms of an independent set of Wilson loops. The final solution for the independent degrees of freedom is surprisingly simple: it suffices to use the Wilson loops of small lattice loops, consisting of no more than 6 lattice links. - At this point it may be noted that a similar problem arises in 2+1-gravity (in the connection formulation) on a manifold $\Sigma^{g} \times \mathbb{R}$, with a compact, two-dimensional Riemann surface $\Sigma^{g}$ of genus $g$. One also has a discrete set of loops, namely, the elements of the homotopy group of $\Sigma^{g}$, and can introduce the corresponding Wilson loops. Again, one is interested in finding a maximally reduced set of such Wilson loops, in terms of which all the others can be expressed, using the Mandelstam constraints (see, for example, Nelson and Regge (1991), Ashtekar and Loll (1994)).

Of course it does not suffice to establish a set of independent loop variables on the lattice; one also has to show that the kinematics can be expressed in a manageable form in terms of these variables. Some important problems are the following. 
a) Since the space $\mathcal{C}_{\text {phy }}=\mathcal{A} / \mathcal{G}$ is topologically non-trivial, the set of independent lattice Wilson loops cannot provide a good global chart of $\mathcal{C}_{\text {phy }}$. It would therefore be desirable to find a minimal embedding into a space of loop variables that is topologically trivial (together with a finite number of non-linear constraints that define the space $\mathcal{C}_{\text {phy }}$ ).

b) What is the explicit form of the Jacobian of the transformation from the holonomy link variables to the Wilson loops? From this one could derive an effective action/Hamiltonian for gauge theory, and maybe derive a prescription of how to translate local quantities into their loop space analogues.

c) Is it possible to define a perturbation theory in loop space, once one understands which Wilson loop configurations contribute most in the path integral, say? This would be an interesting alternative to the usual perturbation theory in $A$ and the $\frac{1}{N}$-expansion, and may lead to new and more efficient ways of doing calculations in lattice gauge theory.

d) Lastly, in finding a solution to the Mandelstam constraints it was crucial to have the concept of a smallest loop size on the lattice (the loops of link length 4 going around a single plaquette). What are the consequences of this result for the continuum theory, where there is no obstruction to shrinking loops to points?

Some progress on (a) and (b) has been made for small lattices Loll (1993a), and it is clear that all of the above-posed problems are rather non-trivial, even in the discretized lattice theory where the number of degrees of freedom is effectively finite. One may be able to apply some of the techniques used here to 3+1-dimensional gravity, where also discrete structures appear (the generalized knot classes) after factoring out by the spatial diffeomorphisms.

Attempts to apply lattice gauge theory ideas directly to general relativity have so far failed, basically because the diffeomorphism invariance of the theory is "very badly broken" by the introduction of a lattice in space. This finds expression in the fact that the algebra of constraints does not close on the lattice, and little is known about whether closure can be achieved in the continuum limit as the lattice spacing goes to zero (Renteln 1990). Nevertheless, some lattice-inspired ideas have made their way into gravity. In these cases, the lattice is not to be thought of as a fixed hypercubic lattice imbedded into space, but defined abstractly through its connectivity properties, i.e. a list of data of how pieces of paths in $\Sigma$ link together to form a generalized lattice or graph. These are properties that are invariant under diffeomorphisms. Using such ideas, one may construct diffeomorphism-invariant measures on the space $\mathcal{A}$ of connections (Ashtekar and Lewandowski 1993b, Baez 1993). 


\section{Quantization in the loop approach}

I will now return to the discussion of the continuum theory of both gravity and gauge theory, and review a few concepts that have been used in their quantization in a loop formulation. The main idea is to base the canonical quantization of a theory with configuration space $\mathcal{A}$ on the non-local, gauge-invariant phase space variables $T(\gamma)$ (and not on some gaugecovariant local field variables). This is a somewhat unusual procedure in the quantization of a field theory, but takes directly into account the non-linearities of the theory.

You may have noted that so far we have only talked about configuration space variables, the Wilson loops. For a canonical quantization we obviously need some momentum variables that depend on the generalized electric field $\tilde{E}$. Since $\tilde{E}$ - unlike the gauge potential $A$ - is not a one-form, we cannot introduce holonomy variables that depend only on $\tilde{E}$. This leads to a certain asymmetry in the formulation. One choice of a generalized Wilson loop that also depends on $\tilde{E}$ is

$$
T_{(A, \tilde{E})}^{a}(\gamma, s):=\operatorname{Tr} U_{\gamma}(s) \tilde{E}^{a}(\gamma(s))
$$

This variable depends now on both a loop and a marked point, and it is still gauge-invariant (under the transformations (2.3)). Also it is strictly speaking not a function on phase space, but a vector density. This latter fact may be remedied by integrating $T^{a}$ over a twodimensional "ribbon" or "strip", i.e. a non-degenerate one-parameter congruence of curves $\gamma_{t}(s)=: R(s, t), t \in[0,1]$ (Ashtekar and Isham 1992). In any case, the "momentum Wilson loop" depends not just on a loop, but needs an additional geometric ingredient. I will use here the unsmeared version since it does not affect what I am going to say.

The crucial ingredient in the canonical quantization is the fact that the loop variables $\left(T(\gamma), T^{a}(\gamma, s)\right)$ form a closing algebra with respect to the canonical symplectic structure on $T^{*} \mathcal{A}$. This fact was first established by Gambini and Trias (1986) for gauge group $S U(N)$ and later rediscovered by Rovelli and Smolin (1990) in the context of gravity. For the special case of $G=S L(2, \mathbb{C})$ (or any of its subgroups) in the fundamental representation, the Poisson algebra of the loop variables is

$$
\begin{aligned}
& \left\{T\left(\gamma_{1}\right), T\left(\gamma_{2}\right)\right\}=0, \\
& \begin{aligned}
&\left\{T^{a}\left(\gamma_{1}, s\right), T\left(\gamma_{2}\right)\right\}=-\Delta^{a}\left(\gamma_{2}, \gamma_{1}(s)\right)\left(T\left(\gamma_{1} \circ_{s} \gamma_{2}\right)-T\left(\gamma_{1} \circ_{s} \gamma_{2}^{-1}\right)\right) \\
&\left\{T^{a}\left(\gamma_{1}, s\right), T^{b}\left(\gamma_{2}, t\right)\right\}=-\Delta^{a}\left(\gamma_{2}, \gamma_{1}(s)\right)\left(T^{b}\left(\gamma_{1} \circ_{s} \gamma_{2}, u(t)\right)+T^{b}\left(\gamma_{1} \circ_{s} \gamma_{2}^{-1}, u(t)\right)\right)+ \\
&+\Delta^{b}\left(\gamma_{1}, \gamma_{2}(t)\right)\left(T^{a}\left(\gamma_{2} \circ_{t} \gamma_{1}, v(s)\right)+T^{a}\left(\gamma_{2} \circ_{t} \gamma_{1}^{-1}, v(s)\right)\right)
\end{aligned}
\end{aligned}
$$


In the derivation of this semi-direct product algebra, the Mandelstam constraints (5.1e) have been used to bring the right-hand sides into a form linear in $T$. The structure constants $\Delta$ are distributional and depend just on the geometry of loops and intersection points,

$$
\Delta^{a}(\gamma, x)=\oint_{\gamma} d t \delta^{3}(\gamma(t), x) \dot{\gamma}^{a}(t)
$$

The Poisson bracket of two loop variables is non-vanishing only when an insertion of an electric field $\tilde{E}$ in a $T^{a}$-variable coincides in $\Sigma$ with (the holonomy of) another loop. For gauge group $S U(N)$, the right-hand sides of the Poisson brackets can no longer be written as linear expressions in $T$.

By a "quantization of the theory in the loop representation" we will mean a representation of the loop algebra (7.2) as the commutator algebra of a set of (self-adjoint) operators $\left(\hat{T}(\gamma), \hat{T}^{a}(\gamma, s)\right.$ ) (or an appropriately smeared version of the momentum operator $\hat{T}^{a}(\gamma, s)$ ). Secondly, the Mandelstam constraints must be incorporated in the quantum theory, for example, by demanding that relations like (5.1) hold also for the corresponding quantum operators $\hat{T}(\gamma)$ (for the momentum Wilson loops there are corresponding Mandelstam constraints).

The completion of this quantization program is different for gauge theory and gravity, in line with the remarks made in section 2 above. For the Yang-Mills theory, one has to find a Hilbert space of wave functionals that depend on either the Wilson loops $T(\gamma)$ or directly on the elements $\gamma$ of some appropriate (quotient of a) loop space. The quantized Wilson loops must be self-adjoint with respect to the inner product on this Hilbert space, since they correspond to genuine observables in the classical theory. Then the Yang-Mills Hamiltonian (YM3) has to be re-expressed as a function of Wilson loops, and finally, one has to solve the eigenvector equation

$$
\hat{H}_{Y M}\left(T(\gamma), T^{a}(\gamma)\right) \Psi(\alpha)=E \Psi(\alpha)
$$

to obtain the spectrum of the theory. Because of the lack of an appropriate scalar product in the continuum theory, the only progress that has been made along these lines is in the lattice-regularized version of the gauge theory (see, for example, Brügmann (1991), Gambini and Setaro (1993)).

For the case of gravity one does not require the loop variables $\left(T(\gamma), T^{a}(\gamma, s)\right)$ to be represented self-adjointly, because they do not constitute observables on the classical phase 
space. At least one such representation (where the generalized Wilson loops are represented as differential operators on a space of loop functionals $\tilde{\Psi}(\gamma)$ ) is known, and is described in Brügmann's article in these proceedings. The completion of the loop quantization program for gravity requires an appropriate inclusion of the three-dimensional diffeomorphism constraints $C_{a}$ and the Hamiltonian constraint $C$, c.f. (GR2), which again means that they have to be re-expressed in terms of loop variables. Then, solutions to these quantum constraints have to be found, i.e. loop functionals that lie in their kernel,

$$
\hat{C}_{a}\left(T(\gamma), T^{a}(\gamma)\right) \Psi(\alpha)=0 \wedge \hat{C}\left(T(\gamma), T^{a}(\gamma)\right) \Psi(\alpha)=0
$$

Finally, the space of these solutions has to be given a Hilbert space structure to make any physical interpretation possible. No appropriate scalar product is not known at the moment, but there exists a large number of solutions to the quantum constraint equations (7.5), which are related to knot invariants.

There is another interesting mathematical structure in the loop approach which I would like to mention here, which tries to establish a relation between the quantum connection and the quantum loop representation(s). This is the so-called loop transform, first introduced by Rovelli and Smolin, which is supposed to intertwine the two types of representations according to

$$
\tilde{\Psi}(\gamma)=\int_{\mathcal{A} / \mathcal{G}}[d A]_{\mathcal{G}} h[\gamma, A] \Psi_{\text {phy }}(A)
$$

and

$$
(\hat{T}(\alpha) \tilde{\Psi})(\gamma):=\int_{\mathcal{A} / \mathcal{G}}[d A]_{\mathcal{G}} h[\gamma, A]\left(\hat{T}(\alpha) \Psi_{\text {phy }}(A)\right)
$$

and similarly for the action of $\hat{T}^{a}(\gamma)$. Indeed, it was by the heuristic use of (7.6) and (7.7) that Rovelli and Smolin arrived at the quantum representation of the loop algebra (7.2) mentioned above. The relation (7.6) is to be thought of as a non-linear analogue of the Fourier transform

$$
\tilde{\Psi}(p)=\frac{1}{\sqrt{2 \pi}} \int d x e^{i x p} \Psi(x)
$$


on $L^{2}(\mathbb{R}, d x)$, but does in general not possess an inverse. Again, for Yang-Mills theory we do not know how to construct a concrete measure $[d A]_{\mathcal{G}}$ that would allow us to proceed with a loop quantization program, although in this case the loop transform can be given a rigorous mathematical definition.

For the application to gravity, the domain space of the integration will be a smaller space $\mathcal{M}$, as explained in Section 2 above, but for this case we know even less about the well-definedness of expressions like (7.6) and (7.7). There is however a variety of simpler examples, such as 2+1-dimensional gravity (Ashtekar et al. 1989, Ashtekar and Loll 1994) and electrodynamics (Ashtekar and Rovelli 1992), where the loop transform is a useful and mathematically well-defined tool.

\section{Outlook}

In this article I have reviewed various aspects of the loop formulation of gauge theory and gravity, and explained in some detail the motivation and justification for adopting such an approach in place of a formulation in terms of local field variables. An important aspect of the loop formulation is its constrained character, due to the presence of the Mandelstam constraints. There is as yet no complete proposal for a loop formulation of any non-linear quantum field theory with local degrees of freedom, like the cases of general relativity and Yang-Mills theory we have been considering. The instances where such a program can be completed are typically of topological type, and their reduced phase spaces are finite-dimensional (examples are $2+1$ gravity and $1+1$ Yang-Mills theory).

It has to be kept in mind that any such proposal involves a number of non-trivial choices along the way, which will consist roughly of the following:

- a choice of an initial space of loops and a set of functions on it corresponding - after imposition of a complete set of constraints - to the physical configurations of the system. These choices are presumably different for the classical and quantum theories.

- A differential calculus on the space of loop functions, specifying the nature of differential operators, and conditions under which differential equations possess solutions and whether those solutions are unique.

- A prescription of how equations of motion are to be derived: whether from some action principle on the space of loop functions or by translating (e.g. by using a "loop transform") expressions from the connection formulation. 
- A choice of a (unitary irreducible) representation of an algebra of loop functions in the quantum theory, like the T-algebra of Gambini and Trias or some variation of it. If such representations exist, they are likely to be non-unique, in which case additional physical criteria will be needed to narrow down the choice as much as possible.

- A prescription of how the Mandelstam constraints and inequalities are to imposed on either the classical or the quantum level.

- An appropriate choice of regularization and, if necessary, renormalization of loop space operators; in the case of gravity, this choice should be "as diffeomorphism-invariant as possible".

At this stage we do not have a good description of the set of all such choices, and it is therefore hard to tell if specific choices that have been adopted in ongoing research programs are very special or in fact quite generic and natural. Moreover, for at least some of the above points, the correct choices are likely to be different for gauge theory and general relativity. One hope for the solution of the latter is that the requirement of diffeomorphism invariance may come to our help, because it naturally leads to discrete geometric stuctures which may be easier to handle mathematically. - There still is much to be explored in this most fascinating line of research!

Acknowledgement. I would like to thank the organizers, in particular H. Friedrich, for their hospitality and efforts to make this school as well-coordinated as possible. 


\section{References}

Anandan, J. (1983): Holonomy groups in gravity and gauge fields, in Proceedings of the Conference on Differential Geometric Methods in Theoretical Physics, Trieste 1981, eds. G. Denardo and H.D. Doebner, World Scientific

Aref'eva, I.Ya. (1979): The gauge field as chiral field on the path and its integrability, Lett. Math. Phys. 3, 241-247

Arms, J.M., Marsden, J.E. and Moncrief, V. (1981): Symmetry and bifurcations of momentum mappings, Comm. Math. Phys. 78, 455-478

Ashtekar, A. (1991): Lectures on non-perturbative gravity, World Scientific, Singapore

Ashtekar, A. (1993): Mathematical problems of non-perturbative quantum general relativity, in: Proceedings of the 1992 Les Houches summer school on gravitation and quantization, ed. B. Julia, North-Holland, Amsterdam

Ashtekar, A., Husain, V., Rovelli, C., Samuel, J. and Smolin, L. (1989): $2+1$ gravity as a toy model for the $3+1$ theory, Class. Quan. Grav. 6, L185-193

Ashtekar, A. and Isham, C.J. (1992): Representations of the holonomy algebras of gravity and non-Abelian gauge theories, Class. Quan. Grav. 9, 1433-1467

Ashtekar, A. and Lewandowski, J. (1993a): Completeness of Wilson loop functionals on the moduli space of $S L(2, \mathbb{C})$ and $S U(1,1)$-connections, Class. Quan. Grav. 10, L69-74

Ashtekar, A. and Lewandowski, J. (1993b): Representation theory of analytic holonomy $C^{*}$ algebras, to appear in: Knot theory and quantum gravity, ed. J. Baez, Oxford University Press

Ashtekar, A. and Loll, R. (1994): New loop representations for $2+1$ gravity, Penn State University preprint, in preparation

Ashtekar, A. and Rovelli, C. (1992): A loop representation for the quantum Maxwell field, Class. Quan. Grav. 9, 1121-1150

Baez, $J .(1993)$ : Diffeomorphism-invariant generalized measures on the space of connections 
modulo gauge transformations, to appear in the Proceedings of the Conference on Quantum Topology, eds. L. Crane and D. Yetter, hep-th 9305045

Barrett, J.W. (1991): Holonomy and path structures in general relativity and Yang-Mills theory, Int. J. Theor. Phys. 30, 1171-1215

Bialynicki-Birula, I. (1963): Gauge-invariant variables in the Yang-Mills theory, Bull. Acad. Polon. Sci. 11, 135-138

Brügmann, B. (1991): The method of loops applied to lattice gauge theory, Phys. Rev. D43, $566-579$

Cheng, T.-P. and Li, L.-F. (1984): Gauge theory of elementary particle physics, Clarendon Press, Oxford

Corrigan, E. and Hasslacher, B. (1979): A functional equation for exponential loop integrals in gauge theories, Phys. Lett. 81B, 181-184

Durhuus, B. and Leinaas, J.M. (1982): On the loop space formulation of gauge theories, Physica Scripta 25, 504-510

Fischer, A.E. (1986): A grand superspace for unified field theories, Gen. Rel. Gravit. 18, $597-608$

Fröhlich, J. (1979): Some results and comments on quantized gauge fields, in: Recent developments in gauge theories, Cargèse (ed. 't Hooft et al.)

Gambini, R., Gianvittorio, R. and Trias, A. (1988): Gauge Higgs dynamics in the loop space, Phys. Rev. D38, 702-705

Gambini, R. (1991): Loop space representation of quantum general relativity and the group of loops, Phys. Lett. 255B, 180-188

Gambini, R. and Setaro, L. (1993): SU(2) QCD in the path representation, preprint Montevideo

Gambini, R. and Trias, A. (1981): Geometrical origin of gauge theories, Phys. Rev. D23, $553-555$

Gambini, R. and Trias, A. (1983): Chiral formulation of Yang-Mills equations: A geometric approach, Phys. Rev. D27, 2935-2939 
Gambini, R. and Trias, A. (1986): Gauge dynamics in the C-representation, Nucl. Phys. B278, 436-448

Giles, R. (1981): Reconstruction of gauge potentials from Wilson loops, Phys. Rev. D24, 2160-2168

Gliozzi, F. and Virasoro, M.A. (1980): The interaction among dual strings as a manifestation of the gauge group, Nucl. Phys. B164, 141-151

Goldberg, J.N., Lewandowski, J. and Stornaiolo, C. (1992): Degeneracy in loop variables, Comm. Math. Phys. 148, 377-402

Gribov, V.N. (1978): Quantization of non-abelian gauge theories, Nucl. Phys. B139, 1-19

Isham, C.J. (1984): Topological and global aspects of quantum theory, in: Relativity, Groups and Topology II, ed. B.S. DeWitt and R. Stora, North-Holland, Amsterdam, 1059-1290

Kobayashi, S. and Nomizu, K. (1969): Foundations of differential geometry, Vol.1, Interscience, New York

Kogut, J. and Susskind, L. (1975): Hamiltonian formulation of Wilson's lattice gauge theories, Phys. Rev. D11, 395-408

Kuchar̆, K.V. (1993): Canonical quantum gravity, in: Proceedings of the 13th International Conference on General Relativity and Gravitation, ed. C. Kozameh, IOP Publishing, Bristol

Lewandowski, J. (1993): Group of loops, holonomy maps, path bundle and path connection, Class. Quan. Grav. 10, 879-904

Lichnerowicz, A. (1976): Global theory of connections and holonomy groups, Noordhoff International Publishing (French edition published in 1955)

Loll, R. (1992a): Canonical and BRST-quantization of constrained systems, in: Mathematical aspects of classical field theory, eds. M. Gotay, J. Marsden, V. Moncrief, Contemporary Mathematics Series, vol.12, 503-530

Loll, $R$. (1992b): Independent SU(2)-loop variables and the reduced configuration space of SU(2)-lattice gauge theory, Nucl. Phys. B368, 121-142

Loll, R. (1993a): Yang-Mills theory without Mandelstam constraints, Nucl. Phys. B400, 126-144 
Loll, $R$. (1993b): Loop variable inequalities in gravity and gauge theory, Class. Quan. Grav. $10,1471-1476$

Loll, R. (1993c): Lattice gauge theory in terms of independent Wilson loops, Nucl. Phys. B, Proc. Suppl. 30, 224-227

Loll, $R$. (1993d): Chromodynamics and gravity as theories on loop space, Penn State University preprint CGPG-93/9-1, hep-th 9309056

Makeenko, Yu.M. and Migdal, A.A. (1979): Exact equation for the loop average in multicolor QCD, Phys. Lett. 88B, 135-137 (E: 89B (1980) 437)

Makeenko, Yu.M. and Migdal, A.A. (1981): Quantum chromodynamics as dynamics of loops, Nucl. Phys. B188, 269-316

Mandelstam, S. (1962): Quantum electrodynamics without potentials, Ann. Phys. (NY) 19, $1-24$

Mandelstam, S. (1968): Feynman rules for electromagnetic and Yang-Mills fields from the gauge-independent field-theoretic formalism, Phys. Rev. 175, 1580-1603

Mensky, M.B. (1978): Group of parallel transports and description of particles in curved space-time, Lett. Math. Phys. 2, 175-180

Migdal, A.A. (1983): Loop equations and 1/N expansion, Phys. Rep. 102, 199-290

Mostow, M.A. and Shnider, S. (1983): Does a generic connection depend continuously on the curvature?, Commun. Math. Phys. 90, 417-432

Nelson, J. E. and Regge, T. (1991): $2+1$ gravity for genus > 1, Comm. Math. Phys. 141, 211-223

Polyakov, A.M. (1979): String representations and hidden symmetries for gauge fields, Phys. Lett. $82 \mathrm{~B}, 247-250$

Pullin, J. (1993): Knot theory and quantum gravity in loop space: a primer, in: Proceedings of the Vth Mexican School of Particles and Fields, ed. J.L.Lucio, World Scientific, Singapore

Pressley, A. and Segal, G. (1986): Loop groups, Clarendon Press, Oxford 
Renteln, P. (1990): Some results of SU(2) spinorial lattice gravity, Class. Quan. Grav. 7, 493-502

Rovelli, C. and Smolin, L. (1990): Loop space representation of quantum general relativity, Nucl. Phys. B331, 80-152

Singer, I. (1978): Some remarks on the Gribov ambiguity, Comm. Math. Phys. 60, 7-12

Tavares, J.N. (1993): Chen integrals, generalized loops and loop calculus, Mathematics preprint, University of Porto

Wilson, K. (1974): Confinement of quarks, Phys. Rev. D10, 2445-2459

Yang, C.N. (1974): Integral formalism for gauge fields, Phys. Rev. Lett. 33, 445-447 